\title{
Complex Elbow Injury With Delayed Ulnar Nerve Dysfunction In Peadiatric Age Group: A Rare Case Report
}

\author{
Authors \\ Sulaiman Sath, Berjina Farooq Naqshi, Adil Bashir Shah, Nissar Shah, Syed Asif Rafiq \\ *Corresponding Author \\ Dr Sulaiman Sath (M.S Orthopaedics) \\ Bone and Joint Hospital (Department of Orthopaedics), GMC Srinagar, India \\ Email:sulaiman185@gmail.com
}

\section{Background}

Fracture dislocation of elbow although is a common presentation but elbow dislocation involving olecranon, medial epicondyle physeal injury with delayed ulnar nerve palsy has rarely been reported.

\section{Introduction}

Elbow dislocations represent $3 \%$ to $6 \%$ of elbow injuries with a peak incidence of 13-14 years ${ }^{1}$. Fracture dislocations of elbow commonly involve medial epicondyle,coroniod process of olecranon, radial head and neck and rarely capitellum or trochlea ${ }^{1,2}$.More Complex injuries like monteggia fracture dislocation and elbow dislocation with medial epicondyle avulsion fracture or with condyle fracture are relatively rare as compared to simple injury patterns ${ }^{1,2}$.We report a rare case of 8 year old boy with dislocated elbow with medial epicondyle avulsion, olecranon fracture and delayed ulnar nerve palsy.

\section{Case}

A 8 year old boy presented to us in emergency department with a history of fall on pointed elbow
8 hours after injury. Examination revealed that elbow was swollen, tender to palpation, painful range of motion with intact distal neural and vascular status. Overlying skin was normal with no breach and there was no evidence of injury to any other part. Past history was not significant. $\mathrm{X}$-rays of involved elbow, anterio-posterior and lateral views demonstrated dislocated elbow joint associated with fracture olecranon and medial epicondyle physeal injury(Fig 1).

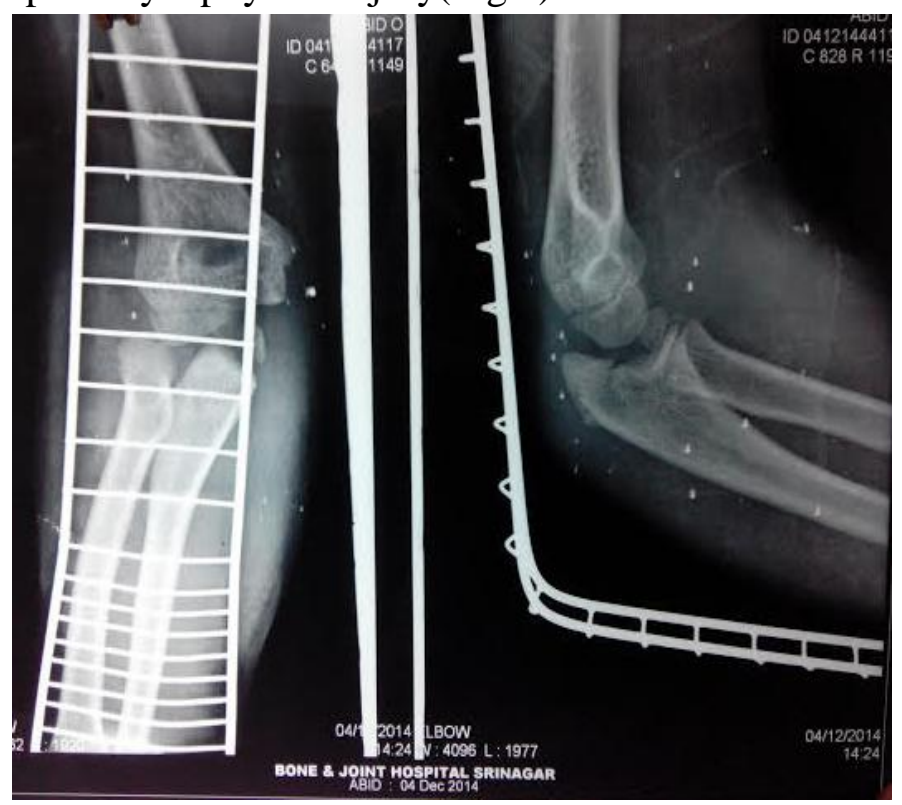

(Fig 1) 
Patient was taken to operating room and closed reduction was done under general anaesthesia which was confirmed under c-arm.After satisfactory reduction, long arm slab was applied which was kept for 3 weeks.Post-operative x-rays were done to check for reduction(Fig2) and neurovascular status was checked which was normal.

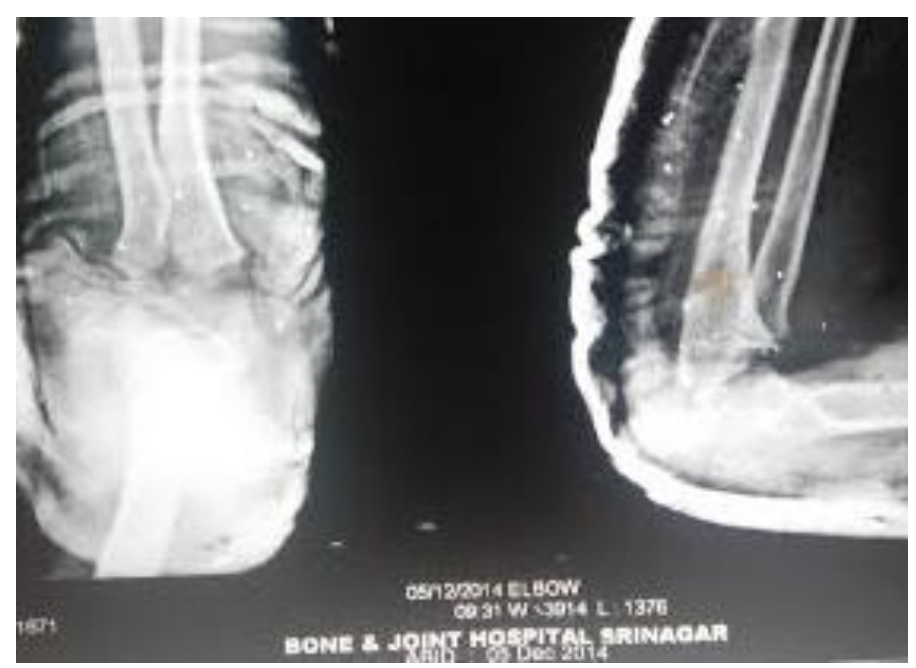

(Fig 2)

After 3 weeks, slab was removed and gentle range of motion exercises were started. When patient returned at 5 weeks followup, he presented with clinical as well as electrophysiological evidence of delayed ulnar nerve dysfunction along with restricted range of motion. X-rays revealed lot of callus on medial side of elbow. Initially conservative treatment was given in the form of physiotherapy, splint and neurotonic medications but patient deteriorated. Ultimately ulnar nerve was explored at 9 weeks after the intial trauma .On exploration via medial approach,ulnar nerve was found entrapped in formed callus of medial epicondyle.Nerve was decompressed of the entrapped callus and was submuscularly transposed anteriorly (Fig3).Postoperatively elbow was immobilized at right angles for 3 weeks. After 3 weeks range of motion was started.Ulnar nerve function recovered over next 2 months and returned to normal. Eventually radiological (Fig 4) as well as clinical
(Fig5) outcome of the patient was excellent with restored distal neurovascular status.

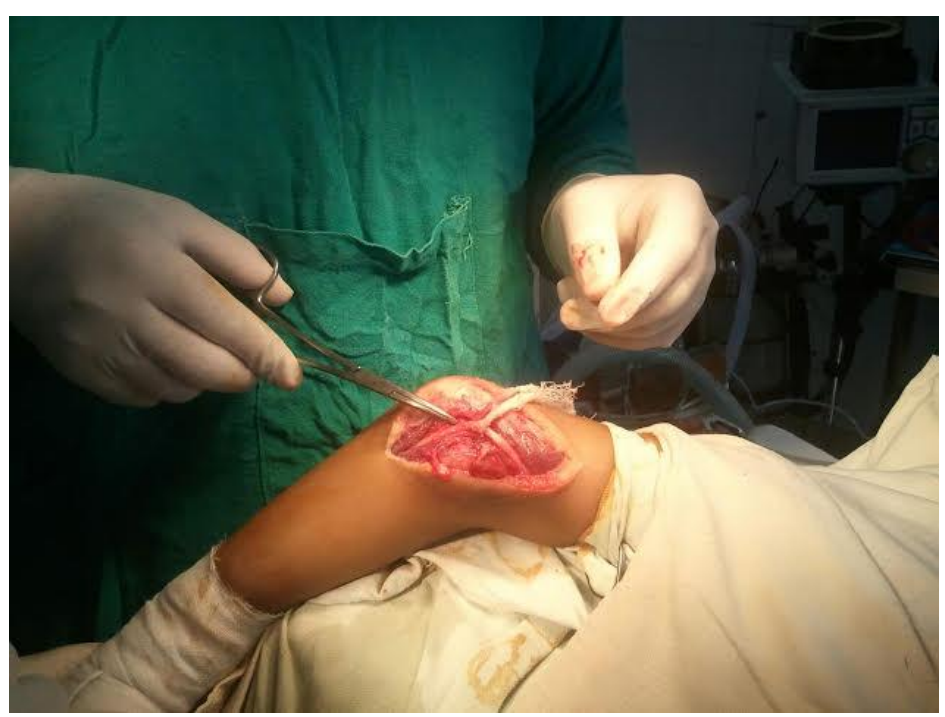

(Fig3)

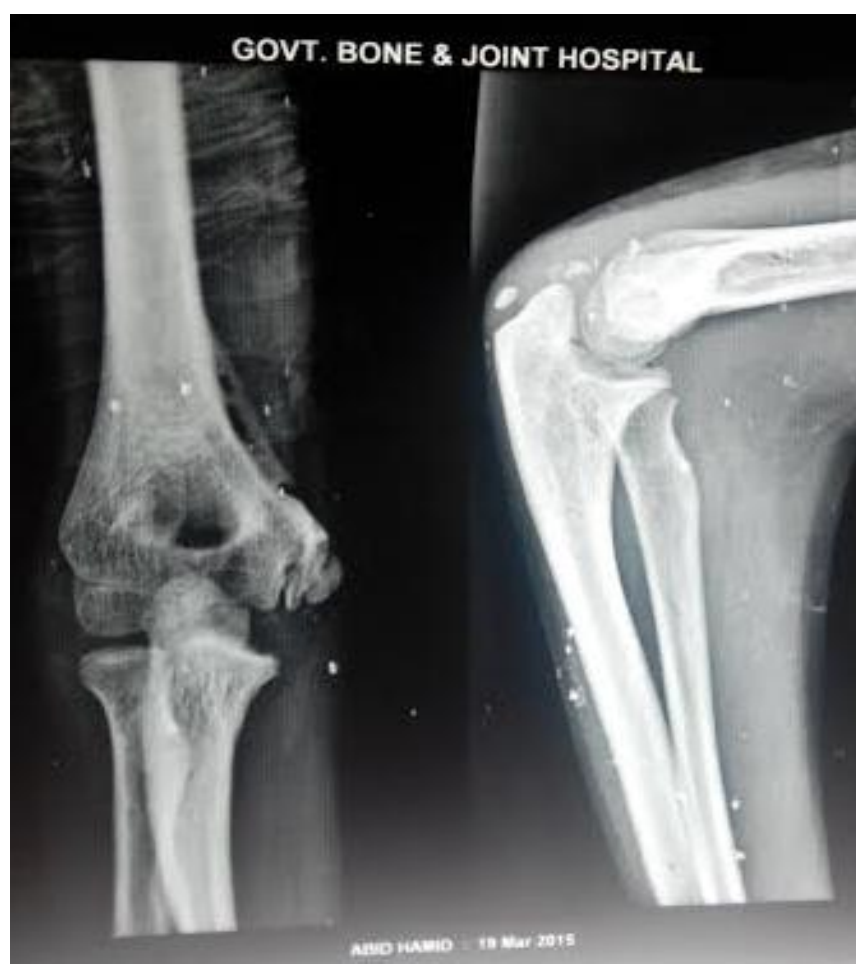

(Fig 4) 

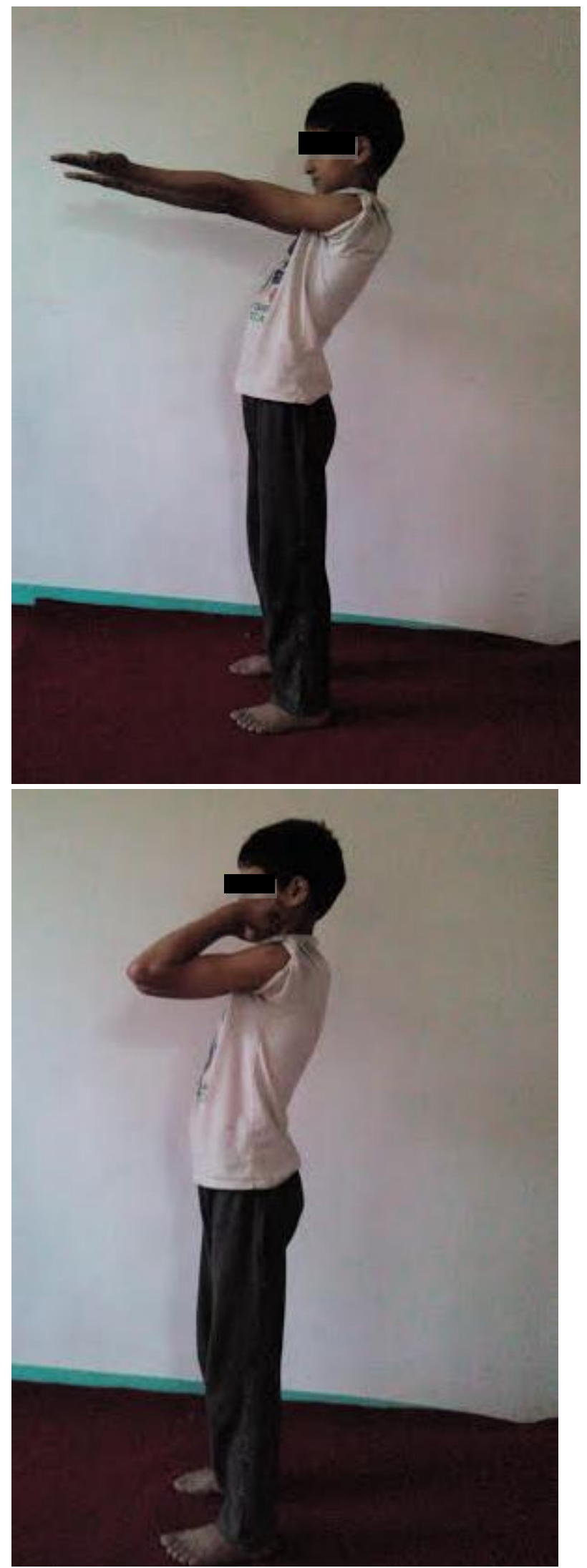

(Fig 5)

\section{Discussion}

Fracture dislocations of elbow in children although is a common occurrence but simultaneous involvement of medial epicondyle and olecranon with late ulnar nerve dsyfunction with elbow dislocation in a single case is quiet rare occurrence and we have not been able to retrieve any such case in litreature .Carlioz and $\mathrm{Abols}^{2}$ et al reported the only series of fracture dislocation of elbow in children and found $64 \%$ have associated fractures or avulsions. They found $36 \%$ of fracture dislocations involving medial epicondyle while only $1.71 \%$ involving olecranon.Rest were involving radial head, neck, lateral flakes of humerus,c oroniod and combined injuries. Ulnar nerve was involved in only $3.44 \%$. Sonawane Darshankumar ${ }^{3}$ et al report a case of extremely complex fracture pattern -elbow subluxation with fracture of the medial epicondyle of humerus with a completely displaced and angulated fracture of the radial neck and fracture of the olecranon in an 8 years male child. He was operated with open reduction and fixation of the fracture medial epicondyle of humerus, closed reduction and fixation of radial neck and the olecranon. The elbow stability was satisfactory and child achieved a good functional result.

Takahito Fujimori ${ }^{4}$ et al reported a rare case of complex elbow injury of 11-year-old Asian boy suffered fracture dislocation elbow after motor vehicle accident. Plain radiographs of the involved elbow showed only a minimally displaced olecranon fracture and a tiny lateral epicondylar avulsion fracture. However, on stress radiographs under general anesthesia revealed severe posterolateral rotatory instability which needed surgical intervention where it was found that the cartilaginous lateral epicondylar apophysis was much larger than the epicondylar fragment on the radiographs and it was after their fixation,elbow stability was regained.

Stavros Ristanis ${ }^{5}$ et al treated three cases of posterolateral elbow dislocation with medial epicondyle fracture in children between 6-8 years by conservative method and found excellent results in all cases by Mayo Elbow Performance Score $X$ rays showed displaced medial epicondyle in two,out of the three cases at at a mean followup of 32.6 months but found no 
clinical correspondence with no deformity or restriction of motion. Fowles ${ }^{6}$ et al reported greater loss of motion of elbow joint in patients who were treated by open method compared to those who were treated by closed method which suggested additiontal trauma of surgery.In their study,here was found to be more soft tissue calcifications in patients treated operatively compared to non-operative ones which reflected more trauma with surgery.

Faierman ${ }^{7}$ et al reported secondary ulnar nerve dsyfunction 1 to 3 months after elbow trauma. They reported two cases who developed features of ulnar nerve dysfunction 4 to 5 weeks after trauma and marked improvement was reported after decompression and transposition of ulnar nerve.

Haflah $\mathrm{NH}^{8}$ et al reported late ulnar nerve palsy in an 11 year old girl two months after fracture dislocation elbow where medial epicondyle injury was missed intially. They found medial epicondyle bony fragment pressing on ulnar nerve intra-operatively which was removed and patient returned to normal activities over period of time.Lima ${ }^{9}$ et al also reported rare case of fracture dislocation elbow involving medial epicondyle which was unrecognized intially and delayed ulnar neuropathy in paediatric age.

In our study, for the fracture dislocation elbow involving olecranon and medial epicondyle because of undisplaced olecranon fracture we did closed reduction which was sucessful and is consistent with the study of Stavros Ristanis ${ }^{5}$ et al which showed excellent results by conservative method.Also Fowles ${ }^{6}$ et al suggested more loss of motion after surgical trauma. Takahito Fujimori ${ }^{4}$ et al in view of instability of lateral column after anaesthesia did surrgical fixation while Sonawane Darshankumar $^{3}$ et al in view of more complex injury did surgical intervention.However, in view of delayed ulnar nerve dsyfunction,surgical decompression and transposition was done and ulnar nerve function returned to normal which is well supported by studies of Faierman ${ }^{7}$ et al and Haflah $\mathrm{NH}^{8}$ et al.

\section{Conclusion}

We conclude that the aim of presenting this rare case report is to keep in mind that the combination of injuries of elbow dislocation,medial epicondyle and olecranon. Also as this patient developed late ulnar nerve dsyfunction so careful evaulation of neurovascular status pre-operatively, postoperatively and each followup should be done.

\section{References}

1. Wilkins KE.Fractures and dislocations of the elbow region.In:Rockwood CA, Wilkins KE,King RE,eds. Fractures in children $\quad 4^{\text {th }} \quad$ Vol.3.Philadelphia: Lipipincott-Raven,1996:653-887.

2. Carlioz H,Abols Y.Posterior dislocation of elbow in children.j Pediatr Orthop 1984;4:8-12.

3. Sonawane D, Gaikwad Y, Khadilkar M. Complex injury of the Elbow in an 8 years Male child - Extreme valgus stress injury: A Case Report. Journal of Trauma \& Orthopaedics. Jan-Mar 2015; 10(1):27-29.

4. Fujimori et al:: Occult posterolateral rotatorydislocation of the elbow with olecranon fracture in a child: a case report. Journal of Medical Case Reports 2012 6:273. . doi:10.1186/1752-1947-6-273.

5. Stavros Ristanis, Haris S. Vasiliadis,Stamatis Mpoulis.Conservative treatment of posterolateral elbow dislocation of the elbow with medial epicondyle fracture in children between 68 years old. Injury Extra .November 2007,vol.38(11) :417-421, doi:10.1016/j. injury.2.2007.02.032.journal ISSN:15723461.

6. J.V. Fowles, N.Slimane ,M.T. Kassab. Elbow dislocation with avulsion of medaial humeral epicondyle.J Bone Joint Surg,Volume 72B, 1990,pp.102-104.

7. 7.Faierman E,Wang J, Jupiter JB. Secondary ulnar nerve palsy in adults after elbow trauma:a report of two cases.J Hand Surg Am. 2001 Jul;26(4):675-8. 
8. Haflah NH,Ibrahim S, Sapuan J,Abdullah S.An elbow dislocation in a child with missed medial epicondyle fracture and late ulnar nerve palsy.J Pediatr Orthop B.2010 Sep;19(5):459-61.

Doi:10.1097/BPB.obo13e32833b6032.

9. Lima S , Correia JF, Ribeiro RP,Martins $\mathrm{RM}$ et al.A rare case of elbow dislocation associated with unrecognized fracture of medial epicondyle and delayed ulnar neuropathy in peadiatric age.J Shoulder Elbow Surg.2013 Mar;22(3):e9-e11. Doi:10.1016/j.jse.2012.11.009. 